
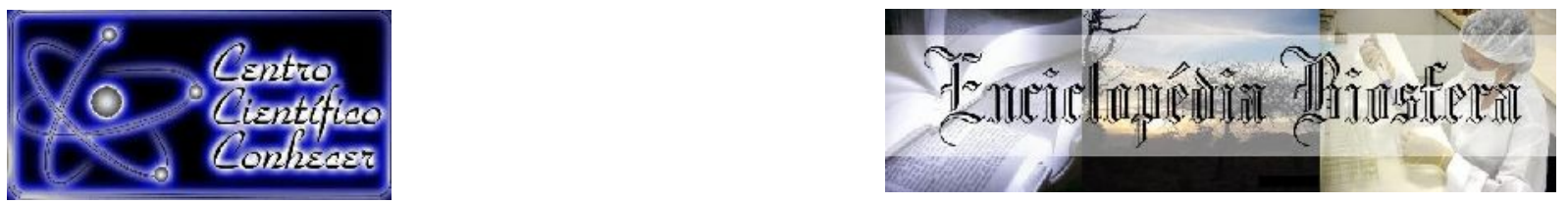

\title{
TRATAMENTO DA TUBERCULOSE: DIFICULDADES ENFRENTADAS POR PACIENTES DE UMA UNIDADE DE SAÚDE DO ACRE
}

\author{
Andréia Maia Cavalcante ${ }^{1}$, Divane Araújo de Souza ${ }^{1}$, Kelline Maia Gadelha ${ }^{1}$, \\ Eder Ferreira de Arruda ${ }^{2}$, Ruth Silva Lima da Costa ${ }^{2}$ \\ 1. Bacharéis em Enfermagem pelo Centro Universitário UNINORTE, Rio Branco-AC, Brasil. \\ 2. Docentes do Centro Universitário UNINORTE, Rio Branco-AC, Brasil. E-mail: \\ ederarrud@gmail.com.
}

Recebido em: 04/10/2019 - Aprovado em: 30/11/2019 - Publicado em: 15/12/2019 DOI: 10.18677/EnciBio_2019B56

\begin{abstract}
RESUMO
A tuberculose é uma doença infectocontagiosa causada pelo Mycobacterium tuberculosis, na qual o tratamento utilizado na atenção básica é o tratamento diretamente observado. O objetivo geral deste estudo foi identificar as principais dificuldades enfrentadas no tratamento diretamente observado da tuberculose em pacientes atendidos em uma unidade de saúde do Acre. Tratou-se de um estudo descritivo realizado com 30 pacientes, nos meses de janeiro a março de 2019, que receberam tratamento para tuberculose em unidade de saúde de referência do município de Rio Branco-Acre. Observou-se que a maioria dos indivíduos diagnosticados se encontravam na faixa etária de 18 a 35 anos $(46,7 \%)$, eram do sexo masculino (63,3\%), com nível superior completo (40,0\%), tinham renda de 1 a 3 salários mínimos $(46,7 \%)$, a forma mais prevalente foi a tuberculose pulmonar $(80,0 \%)$, sem diabetes (83,4\%), não eram tabagistas $(70,0 \%)$, nem etilistas $(76,7 \%)$, não usavam drogas ilícitas $(93,3 \%)$ e não tinham histórico de privação de liberdade $(90,0 \%)$. A maior dificuldade encontrada durante a realização do tratamento foi à reação adversa aos medicamentos $(66,7 \%)$, porém sem dificuldade de acesso ao serviço de saúde $(90,0 \%)$, e possuiam conhecimento sobre as consequências do abandono ao tratamento $(83,3 \%)$. Desse modo, é fundamental que os profissionais e os serviços de saúde conheçam as adversidades enfrentadas pelos pacientes durante o tratamento, pois assim é possível desenvolver ações e estratégias focadas em superar as dificuldades e reduzir a taxa de abandono do tratamento.
\end{abstract}

PALAVRAS-CHAVE: Atenção Básica; Tratamento Farmacológico; Tuberculose.

\section{DIFFICULTIES FACING THE DIRECTLY OBSERVED TREATMENT OF TUBERCULOSIS IN A HEALTH UNIT OF ACRE}

\begin{abstract}
Tuberculosis is an infectious disease caused by Mycobacterium tuberculosis, in which the treatment used in primary care is the treatment directly observed. The general
\end{abstract}


objective of this study is to identify the main difficulties faced in the directly observed treatment of tuberculosis in patients treated at an Acre health unit. This is a descriptive study conducted with 30 patients, from January to March 2019, who were treated for tuberculosis in a referral health unit in the city of Rio Branco-Acre. It was observed that most of the treated individuals were between 18 and 35 years old $(46,7 \%)$, were male $(63,3 \%)$, with complete higher education $(40,0 \%)$, had income 1 to 3 minimum wages $(46,7 \%)$, the most prevalent form was pulmonary tuberculosis $(80,0 \%)$, without diabetes $(83,4 \%)$, neither smokers $(70,0 \%)$, nor alcoholics $(76,7 \%)$, did not use illicit drugs $(93,3 \%)$ and had no history of deprivation of liberty $(90,0 \%)$. The greatest difficulty encountered during treatment was the adverse drug reaction $(66,7 \%)$, but without difficulty in accessing the health service $(90,0 \%)$, and they are aware of the consequences of treatment abandonment $(83,3 \%)$. Thus, it is essential that health professionals and services know the adversities faced by patients during treatment, as it is possible to develop actions and strategies focused on overcoming difficulties and reducing the rate of treatment abandonment.

KEYWORDS: Basic Attention; Pharmacological treatment; Tuberculosis.

\section{INTRODUÇÃO}

A tuberculose (TB) é uma doença infectocontagiosa, tendo como agente etiológico - Mycobacterium tuberculosis (BRASIL, 2011). um terço da população mundial está infectada pelo bacilo de Koch sendo que no Brasil, no ano de 2017, foram notificados 91.000 casos de TB (OPAS, 2018).

A infecção inicia-se quando o $M$. tuberculosis atinge os alvéolos pulmonares causando uma destruição maciça do tecido pulmonar (forma pulmonar) e um pequeno número pode entrar na circulação sanguínea disseminando-se por todo o corpo atingindo outros órgãos e sistemas (forma extrapulmonar), de maneira que ambas as formas podem levar ao óbito caso não haja o tratamento adequado e oportuno (COUTO et al., 2014).

No Brasil, o tratamento da TB na atenção básica é realizado através do tratamento diretamente observado (TDO) que funciona como um importante instrumento para diminuir a resistência aos medicamentos, incentivar a adesão terapêutica e reduzir o abandono. No TDO utiliza-se uma combinação de medicamentos por um período de seis meses. Entretanto, quando ocorre uma interrupção da terapia o protocolo é alterado e são utilizados outros medicamentos que apresentam efeitos colaterais mais acentuados e maior duração no tratamento entre 18 e 24 meses (RABAHI et al., 2017).

Entre os principais fatores que acarretam dificuldade na adesão ao TDO, destacam-se: falta de informação do paciente e da família sobre a doença, uso de álcool e drogas ilícitas, barreiras sociais, econômicas, demográficas e culturais, intolerância medicamentosa, dentre outros (MENDES; FENSTERSEIFER, 2004).

A TB é um importante problema de saúde pública, assim as dificuldades na adesão ao esquema terapêutico podem ocasionar o abandono ou descontinuidade do mesmo, o que pode acarretar maior durabilidade do tratamento, multirresistência bacteriana, além de sofrimento psíquico e social ao paciente. Portanto, é importante identificar os principais problemas enfrentados durante o TDO no intuito de que sejam elaboradas e implantadas intervenções que contribuam para adesão ao tratamento e 
consequente declínio das taxas de morbidade e mortalidade relacionadas a essa patologia (PORTELA et al., 2014).

Dessa forma, o objetivo do presente estudo foi identificar as principais dificuldades enfrentadas no TDO da TB em pacientes atendidos em uma unidade de saúde, no município de Rio Branco Acre.

\section{MATERIAIS E MÉTODOS}

Tratou-se de um estudo observacional do tipo transversal com abordagem quantitativa que foi realizado com pacientes tratados para TB em unidade de saúde de referência do município de Rio Branco-Acre.

Durante os anos de 2017 e 2018, um total de 40 pacientes concluiu o TDO para TB na referida unidade de saúde. Destes, oito não foram localizados e dois não aceitaram participar da pesquisa, dessa forma a amostra do estudo foi composta por 30 indivíduos.

Foram inclusos na pesquisa indivíduos com idade igual ou superior a 18 anos, que concluíram o TDO e que voluntariamente aceitaram a participar das atividades e ações propostas assinando o Termo de Consentimento Livre Esclarecido (TCLE). Deste modo, pacientes com idade inferior a 18 anos, que abandonaram o tratamento ou não aceitaram participar da pesquisa foram excluídos.

A pesquisa foi desenvolvida entre os meses de janeiro a março de 2019, através da aplicação de um questionário sobre as características sociodemográficas, histórico clínico e epidemiológico e dificuldades enfrentadas durante o TDO. O programa estatístico Statistical Package for the Social Sciences (SPSS) versão 21.0 foi utilizado para digitação, revisão e análise dos dados, onde foram calculadas as frequências absolutas e relativas das variáveis de interesse.

A presente pesquisa foi aprovada pelos Comitês de Ética em Pesquisa do Centro Universitário UNINORTE, com o parecer № 3.095 .925 e CAAE № 02794818.2.3001.5009 e do Hospital das Clínicas do Acre - HCA/FUNDHACRE, com o parecer № 3.192.415 e CAAE № 02794818.2.3001.5009.

\section{RESULTADOS E DISCUSSÃO}

A maioria dos indivíduos tratados para TB entre os anos de 2017 e 2018 encontrava-se na faixa etária de 18 a 35 anos (46,7\%), (Tabela 1). Semelhante estudo realizado por Oliveira et al. (2019), na cidade de Recife - PE, no ano de 2017, também verificaram maior frequência de pessoas em tratamento para TB na faixa etária de 18 a 39 anos $(38,9 \%)$. De igual modo, em uma pesquisa desenvolvida por Santos et al. (2018), no estado do Rio de Janeiro, entre os anos de 2011 a 2014, identificaram maior frequência de indivíduos tratados na idade entre 20 a 39 anos $(44,0 \%)$. Segundo Longhi (2013), os adultos jovens têm maior predisposição à progressão da infecção latente para fase clínica da doença.

Com relação ao sexo, 63,3\% dos pacientes eram do sexo masculino (Tabela 1). Bosqui et al. (2017), na cidade de Londrina - PR, encontraram que a maior parte dos casos de TB também ocorreram em homens (76,0\%). Ainda de forma similar, foi observada maior incidência de homens com TB $(63,07 \%)$, em estudo realizado por Silva et al. (2015) entre os anos de 2007 a 2012, no estado do Alagoas. 
Segundo Chaves et al. (2016), os homens são mais vulneráveis à infecção e adoecimento por TB em decorrência de estarem mais suscetíveis aos fatores de risco para doença, tais como: maior presença no mercado de trabalho, menor utilização dos serviços de saúde, maior prevalência de infecção pelo HIV, etilismo e uso abusivo de drogas.

No que diz respeito ao nível de escolaridade, parte considerável dos indivíduos tinham nível superior $(40,0 \%)$, (Tabela 1$)$. De modo divergente, a pesquisa feita por Portela et al. (2014), no estado do Rio de Janeiro, no ano de 2009, revelou que a maioria dos pacientes em tratamento para TB possuíam baixa escolaridade.

Para Orofino et al. (2012), a baixa escolaridade está relacionada à maior frequência de abandono do tratamento, as falhas terapêuticas e a maior mortalidade por TB e isto ocorre, principalmente, em virtude da compreensão e conhecimento ineficaz sobre a doença. Porém, na presente pesquisa foram avaliados indivíduos que concluíram o tratamento para TB, fato que pode justificar a alta porcentagem de pacientes com grau de escolaridade mais elevado.

No que se refere à renda familiar, a maioria dos pacientes tinha renda de um a três salários mínimos (46,7\%), (Tabela 1). Corroborando com esse achado, o trabalho desenvolvido por Couto et al. (2014), através de estudo exploratório, na cidade de João Pessoa-PB, no ano de 2011, também verificaram baixo poder aquisitivo entre os portadores de TB em tratamento, pois segundo estes autores, o poder aquisitivo é um fator determinante para a infecção pelo bacilo de Koch e interfere no tratamento da TB, pois indivíduos com piores condições socioeconômicas estão mais propensos a não concluírem o tratamento.

TABELA 1 - Características sociodemográficas de pacientes tratados para TB em uma unidade de Saúde de Rio Branco, Acre, Brasil, 2017-2018.

\begin{tabular}{ccc}
\hline Variável & $\mathbf{N}$ & $\%$ \\
\hline Faixa etária (anos) & & \\
$18-35$ & 14 & 46,7 \\
$36-45$ & 03 & 10,0 \\
$46-60$ & 06 & 20,0 \\
$>60$ & 07 & 23,3 \\
Escolaridade & & \\
Não alfabetizado & 01 & 3,3 \\
Ensino Fundamental & 08 & 26,6 \\
Ensino Médio & 09 & 30,0 \\
Ensino Superior & 12 & 40,0 \\
Sexo & & \\
Masculino & 19 & 63,3 \\
Feminino & 11 & 36,7 \\
Renda familiar & & 10,0 \\
Nenhuma renda & 03 & 13,3 \\
Até 1 SM & 04 & 46,7 \\
De 1 a 3 SM & 14 & 29,9 \\
\hline 3 SM & 09 & $\mathbf{1 0 0 , 0}$ \\
\hline Total & $\mathbf{3 0}$ & \\
\hline
\end{tabular}


Dados da tabela 2 revelam que a maioria dos indivíduos tinha a TB em sua forma pulmonar (80,0\%). Em um trabalho desenvolvido por Pereira et al. (2015), na cidade de Juiz de Fora-MG, no período de 2008 a 2009, observaram que a maioria dos indivíduos também apresentava a doença na forma pulmonar (80,5\%). Ainda de forma similar, em uma pesquisa realizada por Mendes e Fensterseifer (2004), na cidade de Porto AlegreRS, no período de janeiro a fevereiro de 2003 , verificaram maior taxa de pacientes com a forma pulmonar $(88,0 \%)$.

Segundo Ferri et al. (2014), a TB na forma pulmonar é mais abrangente pelo fato do bacilo já entrar no organismo do indivíduo através das vias aéreas e se implantar primeiramente nos pulmões, porém para que ocorra a forma extrapulmonar da TB são necessárias condições mais específicas.

Com relação ao Diabetes Mellitus (DM), 83,4\% dos pacientes informaram não serem diabéticos (Tabela 2). De forma divergente uma pesquisa realizada por Valenzuela-Jiménez et al. (2017), na cidade do México, no ano de 2016, observaram forte associação entre a tuberculose e a DM.

Segundo Abreu et al. (2017) pacientes portadores de DM têm de 2,44 a 8,33, maior risco de contrair tuberculose do que uma pessoa que não apresente a doença. $A$ relação diabetes-tuberculose requer uma atenção especial, já que a DM interfere no metabolismo dos fármacos utilizados no tratamento da TB, o que pode acarretar maior taxa de abandono e insucesso no tratamento, bem como também maior índice de óbitos.

Sobre o etilismo, a maioria dos indivíduos do presente estudo, relatou não fazer uso de bebida alcoólica (76,7\%), (Tabela 2). Um resultado diferente foi encontrado por Silva et al. (2011), na cidade de Rio de Janeiro - RJ, neste a maioria dos pacientes que foram tratados para TB alegaram fazer uso de álcool. A prática do etilismo é um fator agravante para a não adesão ao tratamento de TB. $O$ alcoolismo exerce influência total sobre o prognóstico e o tratamento da doença, tendo em vista que a tuberculose na forma grave é mais frequente em pacientes que fazem o consumo de álcool (MENDES; FENSTERSEIFER, 2004).

TABELA 2 - Histórico clínico e comportamental de pacientes tratados para TB em uma unidade de Saúde de Rio Branco, Acre, Brasil, 2017-2018.

\begin{tabular}{ccc}
\hline Variável & N & $\%$ \\
\hline Classificação da TB & & \\
Pulmonar & 24 & 80,0 \\
Extrapulmonar & 06 & 20,0 \\
Diabetes mellitus & & \\
Sim & 05 & 16,6 \\
Não & 25 & 83,4 \\
Etilismo & & 23,3 \\
Sim & 07 & 76,7 \\
Não & 23 & 30,0 \\
Tabagismo & & 09
\end{tabular}




\begin{tabular}{ccc} 
Não & 21 & 70,0 \\
Drogas ilícitas & 02 & 6,7 \\
Sim & 28 & 93,3 \\
Não & & \\
Privação de liberdade & 03 & 10,0 \\
Sim & 27 & 90,0 \\
Não & $\mathbf{3 0}$ & $\mathbf{1 0 0 , 0}$ \\
\hline Total &
\end{tabular}

No que diz respeito ao tabagismo (Tabela 2), a maior parte dos pacientes não fazia uso de tabaco (70,0\%). Em pesquisa desenvolvida por Pinto Neto et al. (2013), na Santa Casa de Misericórdia, na Cidade de Vitória-ES, entre o ano de 2010 e 2011, verificaram que $67,3 \%$ dos portadores de TB não eram tabagistas, fato positivo pois o hábito de fumar é um importante risco para a ocorrência da infecção por TB, o tabaco configura um indicativo de crescimento de morbidades e mortalidade pela doença.

Com relação ao uso de drogas ilícitas, a maioria informou que não fazia consumo de substâncias psicoativas (93,3\%), (Tabela 2). Em estudo similar realizado por Oliveira et al. (2019), verificaram que a maioria dos indivíduos não faziam uso de substâncias ilícitas $(61,0 \%)$. Também em outro estudo feito por Bosqui et al. (2017), foi identificado que $77,0 \%$ dos indivíduos tratados para TB não faziam uso de drogas ilícitas. Segundo Mendes e Fensterseifer (2004), o uso de drogas ilícitas predispõe o abandono do tratamento da TB, enquanto que nos pacientes que não fazem uso de substâncias psicoativas a taxa de cura chega a $85,9 \%$.

Com relação à passagem pelo sistema prisional, a maioria dos indivíduos do presente estudo, não tinha histórico de privação de liberdade $(90,0 \%)$ (Tabela 2). Pessoas privadas de liberdade são consideradas um grupo de risco para o adoecimento pela TB, isso é resultante da falta ou da realização irregular do tratamento e da detecção tardia dos casos de resistência nos estabelecimentos prisionais que contribuem para a propagação da TB intramuros e para o insucesso do tratamento (VALENÇA et al., 2016).

No presente estudo verificou-se que todos os pacientes finalizaram o TDO com êxito, este resultado pode estar relacionado ao fato de que a maioria apresentou o baixo consumo de drogas ilícitas, álcool, tabaco e privação de liberdade verificada entre os participantes, configurando um grupo com melhor prognóstico em relação à doença.

Em relação às dificuldades enfrentadas no tratamento da TB, 66,7\% dos pacientes relataram que reações adversas às medicações é a principal limitação encontrada (Tabela 3). Em estudo realizado por Souza e Silva (2010) em um município prioritário no controle da tuberculose em Santa Catarina, no ano de 2010, identificaram os efeitos colaterais das medicações como o principal fator causador de dificuldades durante o tratamento da doença. Ainda de forma similar, em uma pesquisa realizada por Mendes e Fensterseifer (2004) verificaram as reações adversas as medicações como fator limitante ao tratamento e término do mesmo. 
TABELA 3- Principais dificuldades enfrentadas por pacientes tratados para TB em uma unidade de Saúde de Rio Branco, Acre, Brasil, 2017-2018.

\begin{tabular}{ccc}
\hline Variável & $\mathbf{N}$ & $\%$ \\
\hline Dificuldades enfrentadas & & \\
Reações adversas às medicações & 20 & 66,7 \\
Falta de apoio do serviço de saúde & 01 & 3,3 \\
Sintomatologia da doença & 05 & 16,7 \\
Preconceito social & 04 & 13,3 \\
Dificuldade de acesso ao serviço de saúde & & \\
Sim & 03 & 10,0 \\
Não & 27 & 90,0 \\
Conhecimento sobre as consequências do abandono & & \\
ao tratamento & 25 & 83,3 \\
Sim & 05 & 16,7 \\
Não & $\mathbf{3 0}$ & $\mathbf{1 0 0 , 0}$ \\
\hline Total & &
\end{tabular}

As medicações utilizadas para o tratamento de tuberculose têm uma eficácia predominante sobre a $M$. tuberculosis, no entanto podem causar diversos efeitos adversos, tais como: alteração da coloração da urina, coceiras pelo corpo, vermelhidão na pele, aumento do apetite, dores estomacais, náuseas, moleza, fraqueza, diarreia e câimbras. Estes efeitos estão ligados a uma maior taxa de abandono do tratamento, uma vez que ocasionam alto número de hospitalizações e de consultas ambulatoriais e domiciliares (MACIEL et al., 2010).

No que diz respeito ao acesso ao serviço de saúde, 90,0\% dos indivíduos não apresentavam às dificuldades de acesso a unidade de saúde para o tratamento da TB (Tabela 3). Palha et al. (2012) em São Paulo- SP, no período de 2008 a 2009, identificaram que $70,0 \%$ dos indivíduos não tinham dificuldade de se deslocar à unidade durante o tratamento.

Para Viegas et al. (2015) as principais barreiras enfrentadas pelos pacientes no acesso aos serviços de saúde são: dificuldades na marcação das consultas, demora no tempo de espera para o atendimento, falta de especialidade médica e dificuldade no estabelecimento de vínculo entre usuário e profissional da saúde devido à grande rotatividade de profissionais.

No que se refere ao conhecimento acerca das consequências da interrupção do tratamento, $83,3 \%$ dos pacientes tinham ciência do resultado do abandono (Tabela 3). Semelhante a um estudo realizado por Costa et al. (2011) na cidade de Rio Grande-RS, no ano de 2006, foi encontrado maior prevalência de pacientes com conhecimento sobre a adesão e consequências do abandono ao tratamento $(60,0 \%)$.

A adesão do paciente ao tratamento depende do seu conhecimento sobre a patologia, duração do tratamento prescrito, importância da regularidade no uso das medicações e da conscientização sobre as consequências ocasionadas pela interrupção do tratamento, tais como aumento do custo e duração do tratamento, 
resistência bacteriana, sofrimento físico e psicológico e aumento no número de óbitos (OLIVEIRA et al., 2017).

\section{CONCLUSÃO}

De forma positiva os indivíduos do presente estudo concluíram o tratamento diretamente observado para tuberculose, porém a principal dificuldade enfrentada por eles foram as reações adversas das medicações, o que pode levar em alguns casos ao abandono do tratamento.

Dessa forma, é fundamental que os profissionais e os serviços de saúde conheçam as adversidades enfrentadas pelos pacientes durante o TDO, pois assim é possível o desenvolvimento de ações e estratégias focadas em superar as dificuldades e reduzir a taxa de abandono do tratamento.

Dentre as estratégias, destaca-se a importância de manter boa relação com o paciente, passando-Ihe confiança e criando um vínculo durante o tratamento, à divulgação de informações sobre a importância da continuidade e término do tratamento, a disseminação de orientações sobre todas as consequências da interrupção do uso das medicações, mesmo que estas causem reações indesejadas, e também facilitar o acesso ao serviço de saúde aos pacientes de TB.

\section{REFERÊNCIAS}

ABREU, R.G.; SOUSA, A.I.A.; OLIVEIRA, M.R.F.; SANCHEZ, M.N. Tuberculose e diabetes: relacionamento probabilístico de bases de dados para o estudo da associação entre ambas doenças. Epidemiologia e Serviços de Saúde, v.26, n.2, p.359-368, 2017. Disponível em: < http://www.scielo.br/pdf/ress/v26n2/2237-9622-ress-26-0200359.pdf >

BOSQUI, L.R.; SILVA, S.S.; SANFELICE, R.A.; MIRANDA-SAMPLA, M.M.; ALVARENGA, D.S.; LUCAS, B.B. et al. Perfil clínico de pacientes com diagnóstico de tuberculose atendidos no Hospital Universitário de Londrina, Paraná. Semina: Ciências Biológicas e da Saúde, v.38, n.1, p.89-98, 2017. Disponível em: <http://www.uel.br/revistas/uel/index.php/seminabio/article/view/27406/22636>. Doi: 10.5433/1679-0367.2017v38n1p89

BRASIL . Tuberculose na Atenção Primária à Saúde. 2.ed. Brasília: Ministério da Saúde, 2011.

CHAVES, E.C.; CARNEIRO, I.C.R.S.; SANTOS, M.I.P.O.; SARGES, N.A.; GUIMARÃES, D.S.O. Diagnóstico da tuberculose pulmonar em idosos de um hospital universitário no período 2009-2013, Belém, Pará. Revista Brasileira de Ciências do Envelhecimento Humano (RBCEH), v.13, n.3, p.299-308, 2016. Disponível em: <http://seer.upf.br/index.php/rbceh/article/download/6558/pdf/>. Doi: http://dx.doi.org/10.5335/rbceh.v13i2.6558 
COSTA, S.M.; MENDONZA-SASSI, R.A.; TEIXEIRA, T.P.; LEIVAS, V.A.L, CÉZAR-VAZ, M.R. Conhecimento dos clientes com tuberculose pulmonar e seus familiares sobre adesão ao tratamento e fatores associados, no município do Rio Grande (RS). Ciência \& Saúde Coletiva, v. 16, n.1, p.1427-1435, 2011. Disponível em: <http://www.scielo.br/pdf/csc/v16s1/a78v16s1.pdf>

COUTO, D.V.; CARVALHO, R.N.; AZEVEDO, E.B.; MORAES, M.N.; PINHEIRO, P.G.O.D. et al. Fatores determinantes para o abandono do tratamento da tuberculose: representações dos usuários de um hospital público. Saúde Debate, v.38, n.102, p. 572-581, 2014. Disponível em: < http://www.scielo.br/pdf/sdeb/v38n102/0103-1104sdeb-38-102-0572.pdf >

FERRI, A.O.; AGUIAR, B.; WILHELM, C.M.; SCHMIDT, D.; FUSSIEGER, F. et al. Diagnóstico da tuberculose: uma revisão. Revista Liberato, v.15, n.24, p.105-212, $2014 . \quad$ Disponível em: <http://www.liberato.com.br/sites/default/files/arquivos/Revista_SIER/v.\%2015,\%20n.\%2 024\%20(2014)/4\%20-\%20Tuberculose.pdf>

LONGHI, R.M.P. Fatores de risco associados ao desenvolvimento de tuberculose na população urbana do município de Dourados - MS. 2013. 75p. Dissertação do curso de Mestrado em Saúde Pública - Fundação Oswaldo Cruz - FIOCRUZ, Rio de Janeiro, 2013. Disponível em: <https://www.arca.fiocruz.br/handle/icict/24544>

MACIEL, E.L.N.; GUIDONI, L.M.; FAVERO, J.L.; HADAD, D.J.; MOLINO, L.P. et al. Efeitos adversos causados pelo novo esquema de tratamento da tuberculose preconizado pelo Ministério da Saúde do Brasil. Jornal Brasileiro de Pneumologia, v.36, n.2, p.232-238, 2010. Disponível em: < http://www.scielo.br/pdf/jbpneu/v36n2/v36n2a12.pdf >.

MENDES, A.M.; FENSTERSEIFER, L.M. Tuberculose: porque os pacientes abandonam o tratamento? Boletim de pneumologia sanitária, v.12, n.1, p. 25-36, 2004.

OLIVEIRA, L.M.P.O.; CAVALCANTE, S.C.; GARCIA, J.S.; OLIVEIRA, M.A.; ARAÚJOJORGE, T.C. et al. O conhecimento sobre tuberculose entre pacientes de uma comunidade de elevada incidência da doença, na cidade do Rio de Janeiro. In: Encontro Nacional de Pesquisa em Educação em Ciências, 11, 2017, Santa Catarina. Anais. Santa Catarina: Educação em Saúde e Educação em Ciências, 2017. 8p. Disponível em: <http://www.abrapecnet.org.br/enpec/xi-enpec/anais/resumos/R04071.pdf>

OLIVEIRA, S.A.G.; LIMA, C.A.; QUIRINO, E.M.B.; ANDRADE, R.M.L.; LIMA, A.A. et al. Adesão e qualidade de vida em pacientes com tuberculose pulmonar. Revista de enfermagem UFPE on line, v.13, n.3, p.697-706, 2019. Disponível em: 
<https://periodicos.ufpe.br/revistas/revistaenfermagem/article/download/236594/31564>. Doi: https://doi.org/10.5205/1981-8963-v13i03a236594p697-706-2019

OPAS. Organización Panamericana de la Salud. Tuberculosis en las Américas 2018, Washington, D.C.: PAHO, 24p., 2018. Disponível em: < http://iris.paho.org/xmlui/bitstream/handle/123456789/49510/OPSCDE18036_spa?sequ ence $=2 \&$ isAllowed $=y>$.

OROFINO, R.L.; BRASIL, P.E.A.; TRAJMAN, A.; SCHMALTZ, C.A.S.; DALCOLMO, M. et al. Preditores dos desfechos do tratamento da tuberculose. Jornal Brasileiro de Pneumologia, v.38, n.1, p. 88-97, 2012. Disponível em: <http://www.scielo.br/pdf/jbpneu/v38n1/v38n1a13.pdf>.

PALHA, P.F.; SILVA, L.M.C; WYSOCKI, A.D.; ANDRADE, R.L.P.; PROTTI, S.T. et al. Acesso aos serviços de atenção à tuberculose: análise da satisfação dos doentes. Revista da Escola de Enfermagem da USP, v. 46, n. 2, p.342-348, 2012. Disponível em: <http://www.scielo.br/pdf/reeusp/v46n2/a11v46n2.pdf>.

PEREIRA, J.C.; SILVA, M.R.; COSTA, R.R.; GUIMARÃES, M.D.C.; LEITE, I.C.G. Perfil e seguimento dos pacientes com tuberculose em município prioritário no Brasil. Revista de Saúde Pública, v.49, n.6, p. 49-56, 2015. Disponível em: <http://www.scielo.br/pdf/rsp/v49/pt_0034-8910-rsp-S0034-89102015049005304.pdf >. Doi:10.1590/S0034-8910.2015049005304.

PINTO NETO, L.F.S.; VIEIRA, N.F.R.; COTT, F.S.; OLIVEIRA, F.M.A. Prevalência da tuberculose em pacientes infectados pelo vírus da imunodeficiência humana. Revista Brasileira de Clínica Médica, v.11, n.2, p.118-122, 2013. Disponível em: <http://files.bvs.br/upload/S/1679-1010/2013/v11n2/a3563.pdf>.

PORTELA, M.C.; LIMA, S.M.L.; BRITO, C.; FERREIRA, V.M.B.; ESCOSTEGUY, C.C. et al. Programa de Controle da Tuberculose e satisfação dos usuários, Rio de Janeiro. Revista de Saúde Pública, v. 48, n.3, p. 497-507, 2014. Disponível em: < http://www.scielo.br/pdf/rsp/v48n3/pt_0034-8910-rsp-48-3-0497.pdf Doi:10.1590/S0034-8910.2014048004793

RABAHI, M.F.; SILVA JÚNIOR, J.L.R.; FERREIRA, A.C.G.; TANNUS-SILVA, D.G.S.; CONDE, M.B. Tratamento da tuberculose. Jornal Brasileiro de Pneumologia, v.43, n.5, p.472-486, $2017 . \quad$ Disponível em: <http://jornaldepneumologia.com.br/detalhe_artigo.asp?id=2741>. Doi: http://dx.doi.org/10.1590/s1806-37562016000000388

SANTOS, J.N.; SALES, C.M.M.; PRADO, T.N.; MACIEL, E.L. Fatores associados à cura no tratamento da tuberculose no estado do Rio de Janeiro, 2011-2014. Epidemiologia e Serviços de Saúde, v. 27, n.3, 11p., 2018. Disponível em: 
SILVA, C.B.; LAFAIATE, R.S.; DONATO, M. O consumo de álcool durante o tratamento da tuberculose: percepção dos pacientes. Revista Eletrônica Saúde Mental Álcool e Drogas (Edição em Português), v.7. n.1, p.10-17, 2011. Disponível em: <http://pepsic.bvsalud.org/pdf/smad/v7n1/03.pdf>

SILVA, E.G., VIEIRA, J.D.S.; CAVALCANTE, A.L.; SANTOS, L.G.M.L.; RODRIGUES, A.P.R.A. et al. Perfil epidemiológico da tuberculose no estado de Alagas de 2007 a 2012. Ciências Biológicas e da Saúde, v.3, n.1, p.31-46, 2015. Disponível em: $<$ https://periodicos.set.edu.br/index.php/fitsbiosaude/article/view/2352/1506>.

SOUZA, S.S.; SILVA, D.M.G.V. Passando pela experiência do tratamento para tuberculose. Texto \& Contexto - Enfermagem, v.19, n.4, p.636-643, 2010. Disponível em: < http://www.scielo.br/pdf/tce/v19n4/05.pdf >.

VALENÇA, M.S.; POSSUELO, L.G.; CEZAR-VAZ, M.R.; SILVA, P.E.A. Tuberculose em presídios brasileiros: uma revisão integrativa da literatura. Ciência \& Saúde Coletiva, v.21, n.7, p.2147-2160, 2016. Disponível em: <http://www.scielo.br/pdf/csc/v21n7/14138123-csc-21-07-2147.pdf>

VALENZUELA-JIMÉNEZ, H.; MANRIQUE-HERNÁNDEZ, E.F.; IDROVO, A.J. Relação da tuberculose com multimorbidade e redes sociais. Jornal Brasileiro de Pneumologia, v.43, n.1, p.51-53, 2017. Disponível em: <http://www.scielo.br/pdf/jbpneu/v43n1/pt_1806-3713-jbpneu-2016000000075.pdf>. Doi: http://dx.doi.org/10.1590/s1806-37562016000000075

VIEGAS, A.P.B.; CARMO, R.F.; LUZ, Z.M.P. Fatores que influenciam o acesso aos serviços de saúde na visão de profissionais e usuários de uma unidade básica de referência. Saúde e Sociedade, v.24, n.1, p.100-112, 2015. Disponível em: <http://www.scielo.br/pdf/sausoc/v24n1/0104-1290-sausoc-24-1-0100.pdf>. Doi: 10.1590/S0104-12902015000100008 